\title{
MODELLING OF CONTINUOUS STIRRED TANK REACTOR USING ARTIFICIAL INTELLIGENCE TECHNIQUES
}

\author{
Suja Malar, R. M.* \& Thyagarajan, T.* \\ *Department of Electrical and Electronics Engineering, PET Engineering College, \\ Vallioor-627117, Tamil Nadu, India \\ ${ }^{* *}$ Department of Instrumentation Engineering, Madras Institute of Technology Campus, \\ Anna University, Chennai-600044, India \\ E-Mail: rsujamonimalar@yahoo.com
}

\begin{abstract}
Continuous Stirred Tank Reactor System (CSTR) is a typical chemical reactor system with complex nonlinear dynamic characteristics. There has been considerable interest in its state estimation and real time control based on mathematical modelling. However, the lack of understanding of the dynamics of the process, the highly sensitive and nonlinear behaviour of the reactor, has made difficult to develop the precise mathematical modelling of the system. An efficient control of the product concentration in CSTR can be achieved only through accurate model. In this paper, attempts are made to alleviate the modelling difficulties using "Artificial Intelligence" (AI) techniques such as neural, fuzzy and neuro-fuzzy. Simulation results demonstrate the effectiveness of Artificial Intelligence modelling techniques. The performance comparison of different modelling techniques has been given in terms of root mean square error.

(Received in October 2008, accepted in May 2009. This paper was with the authors 2 months for 1 revision.)
\end{abstract}

Key Words: CSTR, Modelling, Neural Networks, Fuzzy, Neuro-Fuzzy

\section{INTRODUCTION}

Developing mathematical models of non-linear systems is a central topic in many disciplines of engineering. Models can be used for simulations, analysis of the system's behaviour, better understanding of the underlying mechanisms in the system, design of new processes and design of controllers. In a control system the plant displaying nonlinearities has to be described accurately in order to design an effective controller. In obtaining the mathematical model, the designer follows two methods. The first one is to formulate the model from first principles using the laws governing the system. This is generally referred to as mathematical modelling. The second approach requires the experimental data obtained by exciting the plant and measuring its response. This is called system identification and is preferred in the cases where the plant or process involves extremely complex physical phenomena or exhibits strong nonlinearities [1].

Obtaining a mathematical model for a complex system is complex and time consuming as it often requires some assumptions such as defining an operating point and doing linearization about that point and ignoring some system parameters, etc. This fact has recently led the researchers to exploit the AI techniques using neural and fuzzy tools in modelling complex systems utilizing solely the input-output data sets.

Artificial Neural Networks and Fuzzy Logic have been increasingly in use in many engineering fields since their introduction as mathematical aids by McCulloch and Pitts in 1943, and Zadeh in 1965 respectively. Being branches of Artificial Intelligence, both emulate the human way of using past experiences, adapting itself accordingly and generalizing. While 
the former has the capability of learning by means of parallel connected units, called neurons, which process inputs in accordance with their adaptable weights usually in a recursive manner for approximation; the latter can handle imperfect information through linguistic variables, which are arguments of their corresponding membership functions. After the introduction of back-propagation algorithm for training multi-layer networks Artificial Neural Networks have found many applications in numerous inter-disciplinary areas [2-3]. On the other hand, FL made a great advance in the mid 1970s with some successful results of laboratory experiments by Mamdani and Assilian [4]. In 1985, Takagi and Sugeno [5] proposed a new rule-based modelling technique using FL. Operating with linguistic expressions, fuzzy logic can use the experiences of a human expert and also compensate for inadequate and uncertain knowledge about the system. On the other hand, ANNs have proven superior learning and generalizing capabilities even on completely unknown systems that can only be described by its inputoutput characteristics. By combining these features, more versatile and robust models, called "Neuro-fuzzy" architectures have been developed, [6-7]. Although fuzzy logic allows one to model a system using human knowledge and experience with if then rules, it is not always adequate on its own. This is also true for ANNs, which only deal with numbers rather than linguistic expressions. This deficiency can be overcome by combining the superior features of the two methods, as is performed in ANFIS architecture introduced by Jang, 1993 [8-15].

In the present work three different models have been developed using three different AI techniques namely, ANN, Fuzzy and Neuro-fuzzy, for the CSTR.

\section{CONVENTIONAL MODEL OF CSTR}

The Continuous Stirred Tank Reactor with single input and single output is shown in Fig. 1. Here isothermal series-parallel reaction (Van der Vusse reaction) is considered to study the steady state and dynamic behaviour of CSTR. The two reactions are:

$$
\begin{aligned}
& A \longrightarrow \underset{K 1}{\longrightarrow} B \longrightarrow \overrightarrow{K 2} C \\
& 2 A \underset{K 3}{\longrightarrow} D
\end{aligned}
$$

A - Cyclopentaddiene, B - Cyclopentenol, C - Cyclopentanediol, D - Dicyclopendtadiene

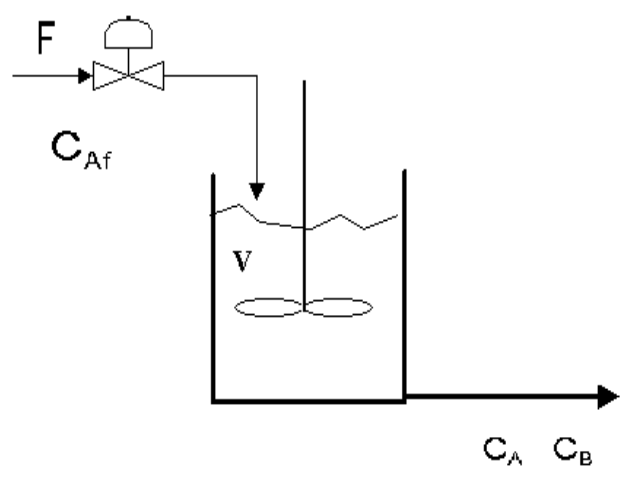

Figure 1: Isothermal CSTR.

The desired product in the reaction is spices $B$, the intermediate product in the primary reaction, which increases the difficulty to control.

The rates of formation of A and B are assumed to be:

$$
\begin{aligned}
& r_{A}=-k_{1}{ }^{c} A-k_{3}{ }^{c} A^{2} \\
& r_{B}=k_{1} C_{A}-k_{2} C_{B}
\end{aligned}
$$


where $k_{1}, k_{2}, k_{3}$ are the reaction rate constants. The feed stream consists of pure A. The mass balances for A and B are given by the following Eq. (4) \& (5):

$$
\begin{gathered}
\frac{d\left(C_{A}\right)}{d t}=\frac{F}{V}\left(C_{A f}-C_{A}\right)-K_{1} C_{A}-K_{3} C_{A}^{2} \\
\frac{d\left(C_{B}\right)}{d t}=-\frac{F}{V} C_{B}+K_{1} C_{A}-K_{2} C_{B}
\end{gathered}
$$

These modelling equations assume a constant volume. The equations for $C_{C}$ and $C_{D}$ are neglected because $C_{B}$ is not dependent on them. The manipulated input in this system is dilution rate. The parameters of the reactor are given in Table I.

Table I: CSTR parameters.

\begin{tabular}{|c|l|}
\hline Parameter & Value \\
\hline$K_{1}$ & $50 \mathrm{~h}^{-1}$ \\
\hline$K_{2}$ & $100 \mathrm{~h}^{-1}$ \\
\hline$K_{3}$ & $1 / 6 \mathrm{~mol} /$ litermin \\
\hline$C_{A f}$ & $10 \mathrm{gmol} /$ liter \\
\hline
\end{tabular}

The steady state equations are:

$$
\begin{gathered}
C_{A s}=\frac{-\left(K_{1}+\frac{F_{s}}{V}\right)}{2 K_{3}}+\frac{\sqrt{\left(K_{1}+\frac{F_{s}}{V}\right)^{2}+4 K_{3}\left(\frac{F_{s}}{V}\right)}}{2 K_{3}} \\
C_{B s}=\frac{K_{1} C_{A s}}{\frac{F_{s}}{V}+K_{2}}
\end{gathered}
$$

These results lead to the steady-state response of the CSTR as shown in the Fig. 2. For an isothermal CSTR steady state input-output can be obtained by relating dilution rate and concentration of component B.

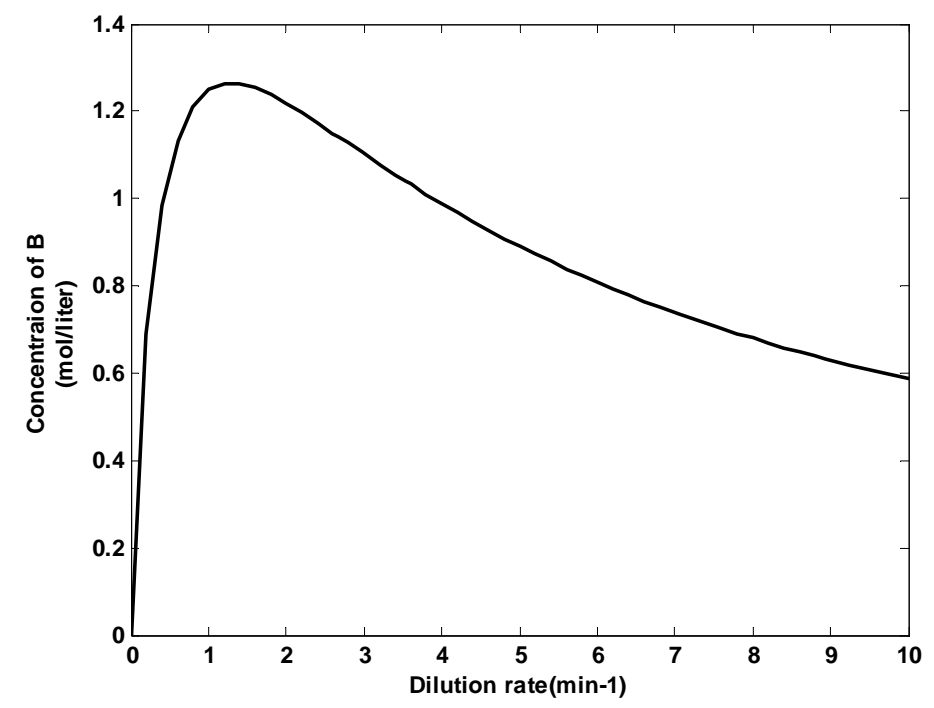

Figure 2: Steady-State response of the CSTR. 
From Fig. 2 it is clear that a nonlinear relationship exists between steady-state dilution rate $(F / V)$ and the steady-state concentration of $\mathrm{B}$. The reactor cannot be controlled at maximum point because the process gain is zero. The steady-state concentration of $\mathrm{B}$ of $1.117 \mathrm{gmol} / \mathrm{liter}$ can be obtained from $F_{s} / V=0.5714 \mathrm{~min}^{-1}$ or $F_{S} / V=2.8744 \mathrm{~min}^{-1}$, showing the presence of input multiplicity.

The Complexity involved in conventional modelling of CSTR increases due to the presence of nonlinearities like input multiplicity, gain sign change, asymmetric response and transformation from minimum to non- minimum phase behaviour and time lag in measuring instruments forcing to make many assumptions, sacrificing the accuracy due to the negligence of uncertainty. Inaccuracy in the modelling due to various assumptions gives degraded performance of controller. In the present work soft computing techniques are used to develop better and more efficient non linear model of CSTR.

\section{NEURAL NETWORK MODELLING}

An artificial neural network is massive, parallel interconnected network of simple elements whose hierarchical are reminiscent of biological neural systems. By looking only at the input and output data a neural network can model non linear systems. ANNs provide an analytical alternative to conventional techniques, which are often limited by strict assumptions of normality, linearity, variable independence etc. The three steps involved in the ANN model development are: 1) Generation of input-output data, 2) Network architecture selection, and 3) Model validation.

\subsection{Generation of input-output data}

The data generated to train the network should contain all the relevant information about the dynamics of the CSTR. The input was given to the conventional model of the CSTR, and from the conventional model, the input and output were sampled for 0.01 sampling instants and the required sampled data are obtained to train the network.

\subsection{Network architecture selection}

The feedforward network topology with sigmoidal activation function was chosen based on the trials with different structures of multilayer perceptron. The variation of error with number of hidden neurons is shown in Fig. 3. The lowest error corresponds to 4 neurons in the hidden layer. Hence it is selected as optimal architecture of ANN. The ANN selected here consists of 3 neurons in the input layer, 4 neurons in the hidden layer and one neuron in the output layer. The ANN architecture used in the present work is shown in Fig. 4.
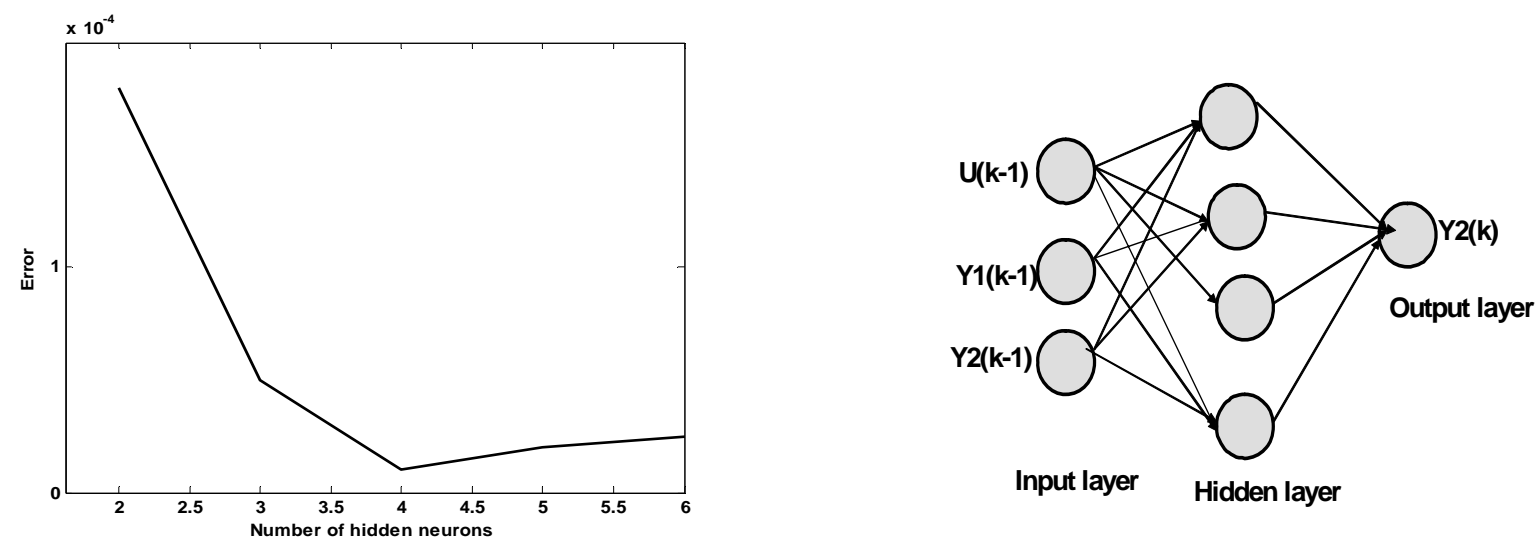

Figure 3: Variation of error with hidden neurons. Figure 4: ANN Model of Isothermal Reactor. 
The training algorithm used in the CSTR modelling is back propagation algorithm. Before training the process weights are initialized to small random numbers. The weights are adjusted till error gets minimized for all training sets. When the error for the entire set is acceptably low, the training is stopped. Table II gives the parameters used in developing the ANN model for the CSTR

Table II: ANN parameters for CSTR modelling.

\begin{tabular}{|l|l|}
\hline Parameters & Value \\
\hline Input Neurons & 4 \\
\hline Output Neurons & 1 \\
\hline Hidden layers & 1 \\
\hline Hidden layer neurons & 5 \\
\hline Activation Function & Sigmoidal \\
\hline Training Algorithm & Levenberg-Marquardt Algorithm \\
\hline Initial Value of $\mu$ & 0.001 \\
\hline$\mu$ decrease factor & 0.1 \\
\hline$\mu$ increase factor & 10 \\
\hline Initial bias & 1 \\
\hline Initial Weights & 1 \\
\hline Iterations & 10000 \\
\hline Architecture & Feed forward \\
\hline
\end{tabular}

\subsection{Model validation}

The final step in developing the model is validation of the model. Validation is performed by evaluating the model performance using trained data and test data. The input and target were presented to the network and the network was trained using Levenburg-Marquardt algorithm. The ANN model was first validated using the trained data which is shown in Fig. 5. The ANN model validation with test data is shown in the Fig. 6. From Figs. 5 and 6 it is seen that there is a perfect overlap between actual output and neural network output.
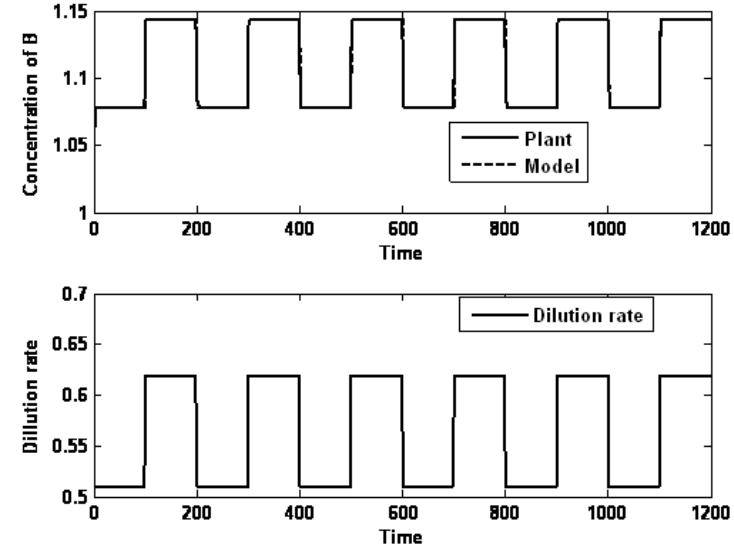

Figure 5: Validation of ANN model with trained data.
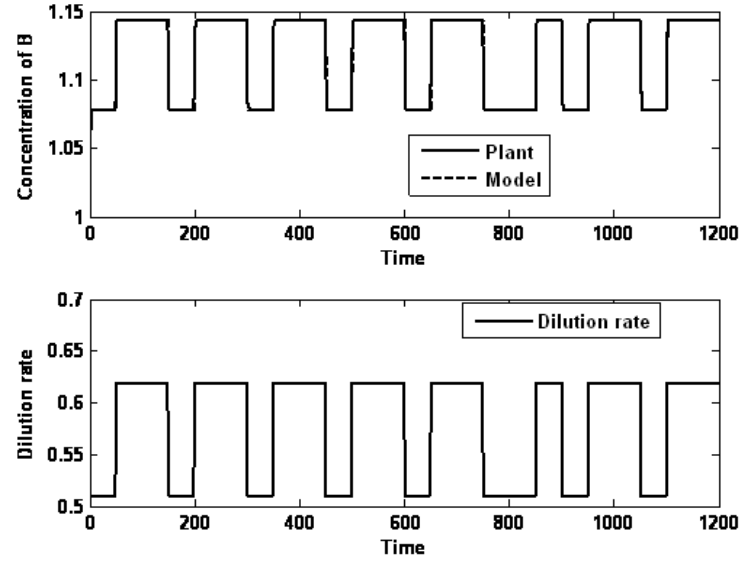

Figure 6: Validation of ANN model with test data.

\section{FUZZY LOGIC MODELLING}

Fuzzy logic provides a practicable way to understand and manually influence the mapping behaviour. In general, fuzzy logic uses simple rules to describe the system of interest, rather 
than analytical equations, making it easy to implement. An advantage, such as robustness and speed, fuzzy logic is one of the best solutions for system modelling and control. The steps involved in the Fuzzy model development are as follows.

\subsection{Selection of input and output variables}

The first stage of fuzzy modelling is the selection of performance variables. Here the input and output variables of the CSTR process are selected. The goal is to find the input variables, which can be best discriminated, and are not affected by noise. The past values of concentration of B component and dilution rate are taken as inputs. The output is value of Concentration of B component. When there is moderate number of input variables in a model, the complexity of the model is reduced.

Possible universe of discourse for the input and output parameters is:

\section{Input parameters}

- Dilution rate $(u(k-1))=0.52$ to 0.62

- Concentration of B component $\left(C_{B}(k-1)\right)=1.02$ to 1.14

- Concentration of A component $\left(C_{A}(k-1)\right)=2.75$ to 3.1

\section{Output parameter}

- Concentration of B component $\left(C_{B}(k)\right)=1.062$ to 1.2

\subsection{Selection of Fuzzy Inference system}

There are two different solutions of fuzzy inference system .Two well known fuzzy modelling methods are the Tuskamoto fuzzy model and Takagi-Sugeno-Kang fuzzy model. In the present work, only TSK model has been considered. TSK fuzzy models are suitable for large class of nonlinear systems.

\subsection{Determination of Number and type of membership functions for each variable}

This choice determines the level of detail (granularity) of the model. Again, the purpose of modelling and the detail of available knowledge will influence this choice. The design of the fuzzy control system starts with establishing certain quantization levels for concentration of B and dilution rate along with membership functions corresponding to these quantization levels. This process defines the appropriate fuzzy sets to be the basis for applying fuzzy logic. These serve as linguistic values to be assigned, respectively, to the fuzzy variables. When the number of fuzzy sets in a input variable is moderate, reasonable labels can be assigned to fuzzy sets. In the present problem five levels for each input and output with Gaussian membership functions are used.

\subsection{Design of rules}

The good adequacy of the identified model allows us to synthesize the control algorithm .In the undertaken model two inputs; and one output, has already been identified. By taking into account the number of membership functions and the constraint associated with CSTR, the fuzzy base containing certain set of rules was designed from the expert knowledge. A Total of 25 fuzzy rules are developed. This control algorithm as fuzzy rules generates geometric representation of the model. When the number of rules is moderate, the overall behaviour of the model is easier to comprehend. This surface is known as control surface and is shown in Fig. 7. 


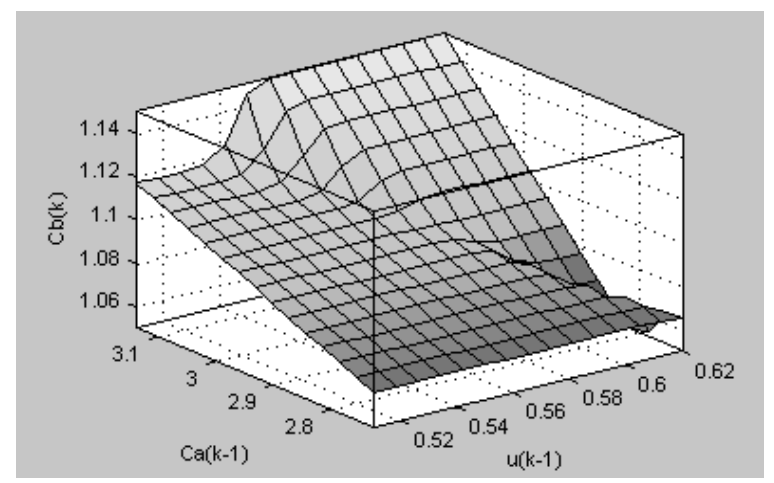

Figure 7: Fuzzy Control surface of CSTR.

\subsection{Fuzzy reasoning}

It is used in a fuzzy rule to determine the rule outcome from the given rule input information. Fuzzy rules represent control strategy or modelling knowledge/experience. When specific information is assigned to input variables in the rule antecedent, fuzzy inference is needed to calculate the outcome for output variable(s) in the rule consequent.

In the first order Sugeno fuzzy model, each rule has a crisp output, the overall output is obtained via weighted average, thus avoiding the time consuming process of defuzzification in Mamdani model. The design parameters of T-S fuzzy inference system are shown in Table III.

Table III: Design parameters of TS-fuzzy inference system.

\begin{tabular}{|c|l|l|}
\hline Sl. No. & Parameter & Value \\
\hline 1 & Input variable & $\begin{array}{l}\text { Present and past values of dilution rate, past value of } \\
\text { concentration of B, A component }\end{array}$ \\
\hline 2 & Quantization levels & 5 \\
\hline 3 & Membership function & Gaussian \\
\hline 4 & Number of rules & 25 \\
\hline 5 & Defuzzification & Centre of sum \\
\hline
\end{tabular}
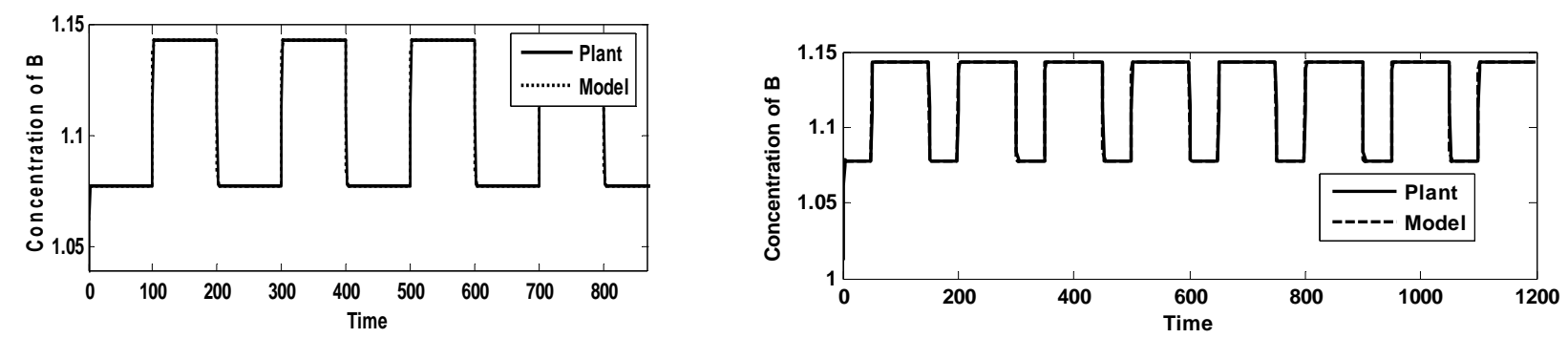

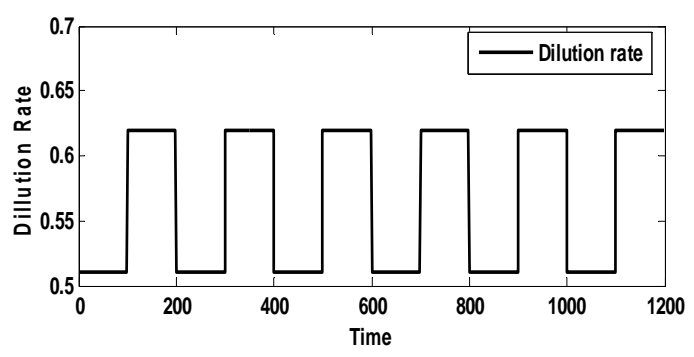

Figure 8: Validation of Fuzzy Model with trained data.

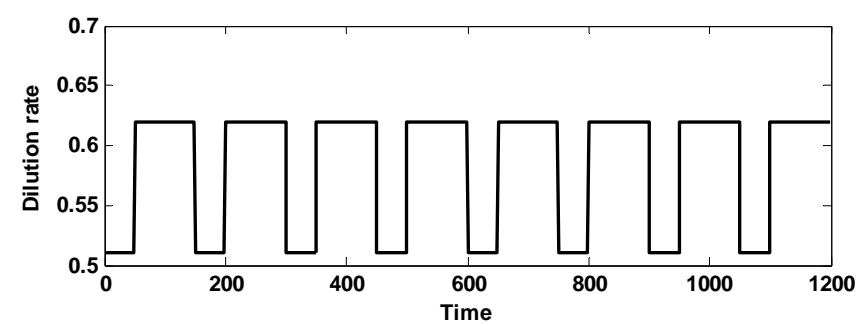

Figure 9: Validation of Fuzzy Model with test data. 


\subsection{Model validation}

The final step of the procedure is validation of the model. Validation is commonly performed by evaluating the model's performance using a data set, which is not shown to the model during the training phase. Five hundred training set data and five hundred validation set data are considered. The validation response is shown in Fig. 8 and Fig. 9.

A fuzzy system of this kind requires a knowledgeable human operator to initialize the system parameters e.g. the membership function bounds. The operator must then optimize these parameters to achieve a required level of accuracy of mapping of the physical system by the fuzzy system. While the visual nature of a fuzzy system facilitates the optimization of the parameters, the need for it to be accomplished manually is a disadvantage.

\section{NEURO FUZZY MODELLING}

ANFIS largely removes the requirement for manual optimization of the fuzzy system parameters. A neural network is used to automatically tune the system parameters, for example the membership function bounds, leading to improved performance without operator invention. In addition to Neural Networks, purely fuzzy approach, an ANFIS was also developed for the estimation of concentration $C_{B}$ because the combination of neural network and fuzzy logic enables the system to learn and improve its performance based on past data. The Neuro-fuzzy system with the learning capability of neural network and with the advantages of the rule-base fuzzy system can improve the performance significantly and can provide a mechanism to incorporate past observations into the classification process. In a neural network the training essentially builds the system. However using a Neuro-fuzzy scheme, the system is built by fuzzy logic definitions and then it is refined using neural network training algorithms.

The three steps involved in the ANFIS model development are: 1) Generation of inputoutput data, 2) Design of Neuro-fuzzy inference system, and 3) Model validation.

\subsection{Generation of input-output data}

The data generated to develop Neuro-fuzzy model should contain all the information about the dynamics of CSTR. The required input-output data were obtained using the conventional model of CSTR through simulation. The input output of Neuro-fuzzy model was shown in Fig. 10.

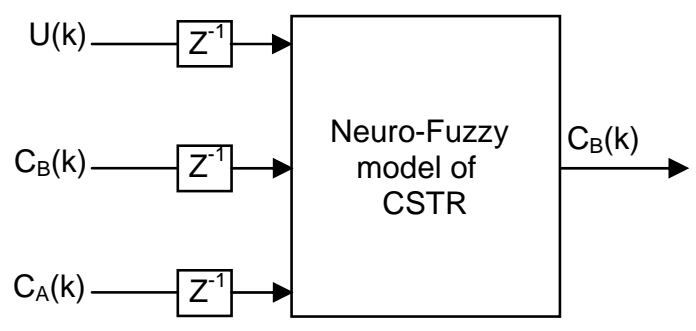

Figure 10: Neuro-fuzzy model of CSTR.

\subsection{Adaptive Neuro-fuzzy inference system design}

A neuro-adaptive learning technique facilitated the learning of information about the data set by the fuzzy modelling procedure, in order to compute the membership function parameters that best allowed the associated FIS to track the given input/output data, rather than choosing the parameters associated with a given membership function arbitrarily. In this study Sugeno 
type FIS have been considered more suitable for constructing fuzzy models due to their more compact and computationally-efficient representation of data than the Mamdani fuzzy systems.

The pre-processed CSTR input/output data which contained all the necessary representative features, was used to train the FIS. The membership function of each input was tuned using the hybrid method consisting of back propagation for the parameters associated with the input membership function and the least square estimation for the parameters associated with the output membership functions. The fuzzy rule architecture of the FIS which consisted of 25 fuzzy rules uses the generalized Gaussian-shaped membership functions.

The computations of the membership function parameters are facilitated by a gradient vector which provides a measure of how well the FIS system is modelling the input/output data. For a given set of parameters, the numbers of nodes in the training data were found to be 286. The numbers of linear parameters and non-linear parameters were found to be 500 and 30 respectively. The hypothesized initial number of membership functions and the type used for each input were 5 and Gaussian respectively. Now, the hypothesized FIS model is trained to emulate the training data by modifying the membership function parameters according to the chosen error criterion. A suitable configuration has to be chosen for the best performance of the network. The number of training epochs was given 100. After the training (with 100 epochs) was complete final configuration for the FIS are given in Table IV. The ANFIS model structure of CSTR is shown in Fig. 11.

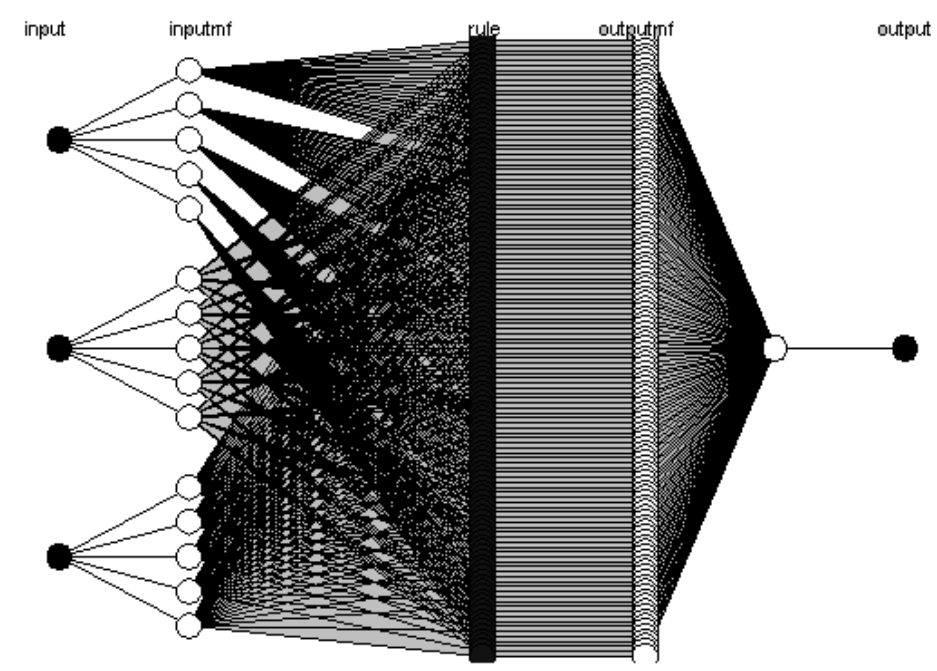

Figure 11: The ANFIS model structure of CSTR.

Table IV: Neuro-fuzzy adaptive network parameters for CSTR modelling.

\begin{tabular}{|l|l|}
\hline Parameter & Value \\
\hline No. of input & 2 \\
\hline No. of membership functions for each input & 5 \\
\hline Type of membership functions for each input & Gaussian \\
\hline No. of rules & 25 \\
\hline Type of membership functions for each output & Linear \\
\hline No. of output membership functions & 25 \\
\hline No. of training epochs & 10 \\
\hline No. of training data sets & 199 \\
\hline No. of testing data sets & 199 \\
\hline
\end{tabular}




\subsection{Model Validation}

The CSTR Neuro-fuzzy model is validated using trained data; the response is shown in Fig. 12. As Fig. 12 clearly shows, the proposed Neuro-fuzzy network is able to accurately model the training data. The comparison of Neuro-fuzzy network predicted outputs to actual outputs is found in the validation plot of test data shown in Fig. 13. As the figure clearly shows, the proposed Neuro-fuzzy network also has the ability to generalize input-output data it was not trained to model.
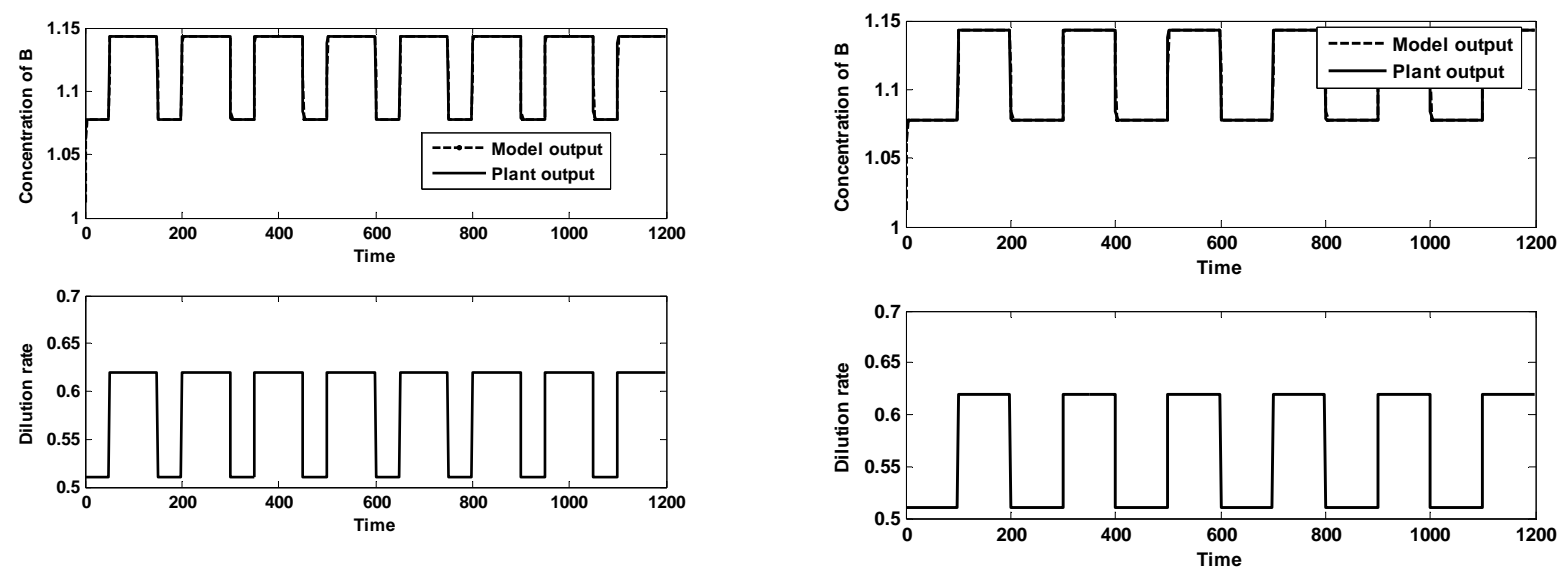

Figure 12: Validation of Neuro-fuzzy Model with trained data.

Figure 13: Validation of Neuro-fuzzy Model with test data.

The ANFIS is a non-linear computational method that has potential for modelling complex systems with unclear input to output relationships due to its ability to combine fuzzy logic and system identification techniques in a hybrid manner. This type of system has several advantages when assigned to applications in which only partial knowledge of the system characteristics is known, as is typically the case with engineering systems. Results from three models are presented in Table $\mathrm{V}$ to access and compare the degree of prediction accuracy and generalization capabilities of the three soft computing techniques modelling of CSTR. The same training and testing data sets were used to train and test three models to extract more solid conclusions from the comparison results. The Neuro-fuzzy model has less training and validation error compared to other artificial intelligence techniques.

Table V: Comparison of different modelling paradigms for CSTR.

\begin{tabular}{|c|c|c|c|c|}
\hline \multirow{2}{*}{$\begin{array}{l}\text { Modelling } \\
\text { Approach }\end{array}$} & \multirow{2}{*}{$\begin{array}{l}\text { Source of } \\
\text { information }\end{array}$} & \multirow{2}{*}{$\begin{array}{l}\text { Method of } \\
\text { acquisition }\end{array}$} & \multicolumn{2}{|c|}{ RMSE } \\
\hline & & & Training & Validation \\
\hline $\begin{array}{l}\text { Conventional } \\
\text { Modelling }\end{array}$ & $\begin{array}{l}\text { Formal data from } \\
\text { first principle }\end{array}$ & $\begin{array}{l}\text { Mathematical } \\
\text { (Lagrange eq.) }\end{array}$ & - & - \\
\hline Neural Modelling & Input-Output data & $\begin{array}{l}\text { Optimization } \\
\text { (learning) }\end{array}$ & 0.0453 & 0.0927 \\
\hline Fuzzy Modelling & Expert knowledge & Knowledge-based & 0.0524 & 0.0854 \\
\hline $\begin{array}{l}\text { Neuro-Fuzzy } \\
\text { Modelling }\end{array}$ & $\begin{array}{l}\text { Input-Output data } \\
\text { and expert } \\
\text { knowledge }\end{array}$ & $\begin{array}{l}\text { Knowledge-based + } \\
\text { learning }\end{array}$ & 0.0343 & 0.04862 \\
\hline
\end{tabular}




\section{CONCLUSION}

It is generally not possible to derive an accurate model of a process or plant especially with nonlinearities. If a reliable model is not available, it is quite difficult to design a controller producing desired outputs. When the data set does not represent the whole operating range adequately, the model to be obtained will not be as robust. Traditional modelling techniques are rather complex and time consuming when we incorporate entire dynamics of the process. However, soft computing techniques namely neural, fuzzy, neuro-fuzzy schemes can approximate the process, using input-output data sets. In the present work, modelling of CSTR was carried out using the above soft computing techniques. The models formulated capture the nonlinearity present in the CSTR. The models thus developed can be used in designing model based control schemes which offers robust controller performance.

\section{REFERENCES}

[1] Patterson, D. W. (1996). Artificial Neural Networks - Theory and Applications, Prentice Hall, India

[2] Engin, S. N.; Gulez, K. (1999). A Wavelet Transform - Artificial Neural Networks (WT-ANN) based Rotating Machinery Fault Diagnostics Methodology, IEEE NSIP '99, Antalya, Turkey, 13

[3] Staszewski, W. J.; Worden, K. (1997). Classification of Faults in Gearboxes - Preprocessing Algorithms and Neural Networks, Neural Computing and applications, Vol. 5, No. 3, 160-183

[4] Mamdani, E. H.; Assilian, S. (1975). An Experiment in Linguistic Synthesis with a Logic Controller, Int. J. Man - Machine Studies, Vol. 8, 1-13

[5] Takagi, S.; Sugeno, M. (1985). Fuzzy identification of fuzzy systems and it's application to modelling and control, IEEE Trans. Systems Man Cybern., Vol. 15, 116-132

[6] Culliere, T.; Titli, A.; Corrieu, J. (1995). Neuro-fuzzy modelling of nonlinear systems for control purposes, Proc. IEEE Int. Conf. on Fuzzy Systems, Yokohama, 2009-2016

[7] Nauck, D. (1994). Fuzzy neuro systems: An overview, Kruse, R.; Gebhardt, J.; Palm, R. (Editors), Fuzzy Systems in Computer Science, 91-107

[8] Jang, J. (1993). ANFIS: Adaptive-Network Based Fuzzy Inference System, IEEE Trans. on Systems, Man and Cybernetics, Vol. 23, No. 3, 665-685

[9] Jang, J.; Sun, C. T. (1995). Neuro-Fuzzy Modeling and Control, IEEE Proc., Vol. 83, No. 3, 378406

[10] Jang, J. (1996). Input Selection for ANFIS Learning, IEEE Fuzzy Systems, 1493-1499

[11] Jang, J. (1996). Neuro-Fuzzy Modeling for Dynamic System Identification, IEEE Fuzzy Systems Symposium, 320-325

[12] Bequette, B. W. (2003). Process Control: Modeling, Design and Simulation, Prentice Hall, Upper Saddle River

[13] Kuure-Kinsey, M.; Cutright, R.; Bequette, B. W. (2006). Computationally efficient neural predictive control based on a feedforward architecture, Ind. Eng. Chem. Res., Vol. 45, No. 25, 8575-8582

[14] Verma, A. K.; Srividiya, A.; Prabhu-Gaonkar, R. S. (2007). Fuzzy-Reability engineering Concepts and Application, Narosa, New Delhi

[15] Gorrostieta, E.; Pedraza, C. (2006). Neuro fuzzy modeling of control systems, Proceeding of the $16^{\text {th }}$ IEEE International Conference on Electronics, Communications and Computers, 23-28 